\title{
Clause du besoin - obstination et mauvais présages
}

Ainsi le Conseil fédéral lance une procédure de consultation concernant l'ordonnance d'exécution de l'art. 55 a LAMal traitant de la clause du besoin. Normalement la durée de cette mesure était limitée à trois ans. Elle expire donc en juillet prochain. Le Parlement s'est laissé convaincre d'autoriser son prolongement pendant une nouvelle période de trois ans, soit jusqu'en 2008. Par souci de «clarification» et de «sécurité juridique», l'ordonnance aimerait limiter à six mois la durée d'une autorisation d'installation. Elle tomberait automatiquement s'il n'en est pas fait usage dans ce délai. Elle n'entend pas prendre en considération le travail à temps partiel pour l'octroi d'une autorisation de pratiquer à charge de l'assurance sociale malgré les promesses faites à cet égard. Elle spécifie que chaque membre d'un cabinet de groupe doit bénéficier d'une autorisation individuelle ... Enfin le message explicatif accompagnant ce projet d'ordonnance se livre à un certain nombre de considérations sur le nombre de médecins qui doivent être admis avec force chiffres et tableaux explicatifs.

Si la FMH concède volontiers que des mesures de régulation sont nécessaires pour gérer la démographie médicale, qu'il s'agisse aussi bien de pléthore que de pénurie, elle n'a jamais souscrit à des modalités aussi sommaires et indifférenciées que la clause du besoin - le Conseil fédéral le sait bien. Cette conception réductrice entre en complète contradiction avec la «concurrence» qu'il essaie de promouvoir par ailleurs. Si le Conseil fédéral était tant soit peu conséquent, il se limiterait à la régulation d'un marché où les partenaires peuvent négocier sur un plan d'égalité en introduisant des paramètres différents de nature à mettre au premier plan la santé publique et l'intérêt général (y compris dans le domaine de la démographie médicale), plutôt que de favoriser grossièrement les payeurs aux dépends des autres, déséquilibrant massivement le tout et créant ainsi une atmosphère conflictuelle peu propice aux solutions.

La prorogation de la clause du besoin jusqu'en 2008 contribue massivement à ces déséquilibres. Elle a et aura encore des effets certains sur le comportement et la stratégie de carrière de nos jeunes collègues. Un rapport de l'OFAS établi en 2004 à la demande du Conseil fédéral l'indique déjà, même si l'expérience n'a été que de deux ans au moment de sa rédaction. Avec six ans de ce régime la déstabilisation aura atteint son comble. Même si la clause du besoin est levée en 2008, la possibilité d'une réintroduction sous une forme ou une autre à la moindre occasion restera dans les esprits. Il sera extrêmement difficile de retrouver la confiance et de compenser les pénuries en train de s'amorcer déjà. Dans ces conditions, limiter la validité des autorisations déjà accordées et ne pas entrer en matière sur la problématique du travail à temps partiel ne fait qu'accentuer encore davantage le caractère grossièrement inapproprié et provocateur de cette mesure. Un mécanisme plus subtil de stabilisation à moyen/long terme assorti de règles du jeu logiques et facilement compréhensibles par tous les intéressés aurait été infiniment préférable. C'est à cela qu'auraient dû servir les trois années écoulées depuis l'introduction abrupte de la clause du besoin. Il n'en a de toute évidence rien été et les signes pointant à l'horizon pour témoigner d'une volonté de limiter la casse et de redresser la barre par une concertation partenariale constructive sont plutôt minces. Ni Priam, ni Hector n'ont jamais voulu entendre Cassandre...

Dr Yves Guisan, Conseiller national, Vice-président FMH 


\section{Zulassungsstopp - Sturheit und böses Omen}

Nun ist es also soweit: Der Bundesrat hat die Verordnung zum Art. 55 a KVG betreffend Zulassungsstopp in die Vernehmlassung geschickt. Eigentlich war die Dauer dieser Massnahme auf drei Jahre beschränkt. Sie würde also im kommenden Juli auslaufen. Doch das Parlament hat sich davon überzeugen lassen, einer Verlängerung um weitere drei Jahre, also bis 2008, zuzustimmen. Im Sinne einer Klarstellung und um Rechtsunsicherheit zu vermeiden, wird in der Verordnung die Dauer der Zulassungsbewilligung auf sechs Monate beschränkt. Sie verfällt automatisch, wenn diese Frist ungenutzt verstreicht. Die Teilzeitarbeit bleibt für den Erhalt einer Zulassungsbewilligung unberücksichtigt - entgegen aller diesbezüglich gemachter Versprechungen. In einer Gruppenpraxis muss jeder einzelne Arzt über eine Praxisbewilligung verfügen ... Zu guter Letzt werden in den Erläuterungen zur Vernehmlassung eine ganze Reihe von Betrachtungen zur Bedarfsplanung angestellt, die mit Zahlen und Grafiken unterlegt sind.

Die FMH ist durchaus auch der Meinung, dass gewisse Massnahmen zur Regulierung der Ärztedemographie - sowohl bei einem Ärzteüberschuss wie einem Ärztemangel - notwendig sind. Einer solch summarischen und undifferenzierten Regelung, wie sie der Zulassungsstopp darstellt, hat die FMH aber nie zugestimmt - das weiss auch der Bundesrat ganz genau. Ein derart einschränkendes Konzept steht in krassem Widerspruch zur «Konkurrenz», der er anderswo so gerne das Wort redet. Wenn der Bundesrat nur ein bisschen konsequent wäre, würde er sich darauf beschränken, in diesen Markt, in dem die Verhandlungspartner gleich lange Spiesse haben, jene Parameter einzuführen, die das Gesundheitswesen und das allgemeine Interesse in den Vordergrund rücken - auch im Bereich der Ärztedemographie. Was er jetzt tut, ist nichts anderes, als die einen Akteure zu Lasten der an- deren auf gröbste Art und Weise zu bevorteilen, das Ganze in ein Ungleichgewicht zu stürzen und damit eine konfliktträchtige Atmosphäre zu schaffen, die Lösungsansätzen abträglich ist.

Die Verlängerung des Zulassungsstopps bis ins Jahr 2008 trägt zu diesem Ungleichgewicht ganz beträchtlich bei. Jetzt schon wirkt sie sich offenkundig auf das Verhalten und die Karriereplanung unserer jungen Kolleginnen und Kollegen aus und sie wird es auch in Zukunft tun. Das zeigt ein 2004 - also nur zwei Jahre nach Verhängen des Zulassungsstopps - vom BSV im Auftrag des Bundesrates erstellter Bericht deutlich auf. Nach sechs Jahren unter diesem Regime wird die Destabilisierung ihren Höhepunkt erreicht haben. Auch wenn der Zulassungsstopp im Jahr 2008 aufgehoben wird: In den Köpfen wird bleiben, dass er beim geringsten Anlass wieder eingeführt werden kann. In einer solchen Situation noch die Dauer der bereits erteilten Berufsausübungsbewilligungen einschränken und auf die Problematik der Teilzeitarbeit nicht einmal eintreten zu wollen, verstärkt nur noch den absolut ungeeigneten und provokativen Ansatz dieser Massnahme. Ein subtilerer, mittel- bis langfristiger Stabilisierungsmechanismus, begleitet von logischen und von allen nachvollziehbaren Spielregeln - wie unendlich viel besser wäre das gewesen! Die vergangenen drei Jahre seit der abrupten Einführung des Zulassungsstopps hätten dafür genutzt werden sollen. Aber ganz offensichtlich ist in dieser Richtung nichts geschehen, und Hoffnungsschimmer am Horizont, durch eine konstruktive und partnerschaftliche Verständigung den angerichteten Schaden vermindern und das Ruder herumreissen zu wollen, sind kaum auszumachen. Aber auch Priam und Hector wollten die Rufe der Cassandra nicht hören...

Dr. med. Yves Guisan, Nationalrat, Vizepräsident FMH 